\title{
Mathematical Modeling and Optimization of the Process of Suberic Binder Obtaining from Outer Birch Bark
}

\author{
Irina G. Sudakova ${ }^{a}$ and Robert Z. Pen ${ }^{b}$ \\ ${ }^{a}$ Institute of Chemistry and Chemical Technology SB RAS \\ 50 Akademgorodok, Krasnoyarsk, 660036, Russia \\ 'Siberian State Technological University \\ 82 Mira, Krasnoyarsk, 660049, Russia
}

Received 09.04.2015, received in revised form 30.04.2015, accepted 12.05.2015

\begin{abstract}
As a result of dispersion analysis of the experimental data the mathematical model alkaline hydrolysis outer birch bark that adequately describes the influence of conditions on the yield and acid number of the suberic binder was obtained. Based on mathematical modeling and solution of the task of process optimization the conditions of alkaline hydrolysis of outer birch bark with a maximum yield of suberic binder $37.8 \mathrm{wt}$. \% have been determined : alkal concentration of $3.1 \%$ by weight .; hydrolysis temperature $88^{\circ} \mathrm{C}$; hydrolysis duration $120 \mathrm{~min}$.
\end{abstract}

Keywords: outer birch bark, suberic binder, dispersion analysis, mathematical modeling and process optimization.

DOI: 10.17516/1998-2836-2015-8-2-256-268.

(C) Siberian Federal University. All rights reserved

* Corresponding author E-mail address: sudakova_irina@mail.ru 


\title{
Математическое моделирование
}

\section{и оптимизация процесса получения суберинового связующего из бересты коры березы}

\author{
И.Г. Судакова ${ }^{a}$, Р.3. Пен ${ }^{6}$ \\ ${ }^{a}$ Институт химии и химической технологии СО РАН \\ Россия, 660036, Красноярск, Академгородок 50, стр. 24 \\ ${ }^{6}$ Сибирский государственный технологический университет \\ Россия, 660049, Красноярск, пр. Мира, 82
}

В результате дисперсионного анализа экспериментальных данных получена математическая модель, адекватно описывающая влияние условий щелочного гидролиза бересты коры березы на выход и кислотное число суберинового связующего. На основании математического моделирования и решения задачи оптимизаџии процесса были определены условия щелочного гидролиза бересты коры березы с максимальным выходом суберинового связующего 37,8\% мас.: концентрация щелочи 3,1\% мас., температура гидролиза $88{ }^{\circ} \mathrm{C}$, продолжсительность гидролиза 120 мин.

Ключевые слова: береста коры березы, субериновое связующее, дисперсионный анализ, математическое моделирование и оптимизация процесса.

\section{Введение}

В настоящее время в мире все большее внимание уделяется повышению экологической безопасности твердых биотоплив. Поэтому получение древесных топливных брикетов с применением нетоксичных связующих является весьма актуальной задачей исследований. Применение природных, экологически чистых связующих позволит улучшить технологические характеристики твердого биотоплива и даст возможность расширить сырьевую базу для его получения [1,2].

Особый интерес представляет получение связующих веществ на основе растительных полимеров [3-5].

Суберин, содержание которого в бересте коры березы составляет от 20 до 40 \% мас., представляет собой природный высокомолекулярный полиэфир. При его гидролизе образуются сложные смеси так называемых субериновых кислот, состав и строение которых определяются способами их извлечения из бересты. Известные способы выделения смесей субериновых веществ основаны на использовании щелочного гидролиза бересты [6-10].

Субериновые кислоты можно использовать в производстве таких технических продуктов, как пленкообразователи, пластификаторы, поверхностно-активные вещества и др. [11-13].

Также этот растительный полимер представляет интерес с точки зрения использования его в качестве связующего при производстве твердых биотоплив [14-16].

Традиционные методы исследования процесса получения суберинового связующего обусловлены экспериментами, которые требуют больших затрат времени и средств, так как они 
основаны на поочередном варьировании отдельных технологических параметров в условиях, когда остальные остаются неизменными.

Целью настоящей работы является проведение дисперсионного анализа экспериментальных данных для определения математической модели, адекватно описывающей влияние условий щелочного гидролиза бересты коры березы на получение суберинового связующего, и нахождение оптимальных условий, обеспечивающих достижение максимально полного выхода суберина.

\section{Экспериментальная часть}

Дисперсионный анализ и математическая обработка результатов выполнены средствами пакета прикладных программ Statgraphics Centurion XVI, блок DOE (Design of Experimental), процедура Malti-Factor Categorical и Excel [17, 18].

В качестве независимых переменных (факторов) были выбраны следующие параметры: $\mathrm{C}$ - концентрация щелочи, \%; Т - температура, ${ }^{\circ} \mathrm{C} ; \mathrm{P}$ - продолжительность, мин. Выходные параметры (результаты процесса): HD - степень гидролиза бересты, \%; V - выход суберина, \%; $\mathrm{K}$ - кислотное число, мг $\mathrm{NaOH} / г$, по которому оценивается содержание поверхностных кислородсодержащих функциональных групп.

Экспериментальные данные, показывающие интервалы варьирования факторов и их влияние на выходные параметры, приведены в объединенной табл. 1.

Таблица 1. Влияние независимых переменных процесса щелочного гидролиза бересты березы на выходные параметры

\begin{tabular}{|c|c|c|c|c|c|}
\hline $\mathrm{C}$ & $\mathrm{T}$ & $\mathrm{P}$ & $\mathrm{HD}$ & $\mathrm{V}$ & $\mathrm{K}$ \\
\hline $\mathbf{1}$ & $\mathbf{2}$ & $\mathbf{3}$ & $\mathbf{4}$ & $\mathbf{5}$ & $\mathbf{6}$ \\
\hline 1 & 60 & 60 & 24,9 & 18,4 & 68 \\
\hline 3 & 60 & 60 & 32,5 & 25,7 & 72 \\
\hline 5 & 60 & 60 & 46,8 & 32,9 & 81 \\
\hline 7 & 60 & 60 & 67,0 & 37,4 & 99 \\
\hline 1 & 90 & 60 & 35,7 & 27,4 & 79 \\
\hline 3 & 90 & 60 & 47,2 & 38,9 & 89 \\
\hline 5 & 90 & 60 & 60,5 & 36,7 & 95 \\
\hline 7 & 90 & 60 & 73,8 & 29,8 & 114 \\
\hline 1 & 120 & 60 & 45,3 & 28,4 & 101 \\
\hline 3 & 120 & 60 & 54,3 & 38,4 & 108 \\
\hline 5 & 120 & 60 & 72,6 & 38,5 & 116 \\
\hline 7 & 120 & 60 & 89,3 & 36,2 & 124 \\
\hline 1 & 90 & 60 & 27,5 & 19,7 & 88 \\
\hline 3 & 90 & 60 & 47,2 & 38,9 & 89 \\
\hline 5 & 90 & 60 & 67,6 & 37,1 & 122 \\
\hline 7 & 90 & 60 & 91,2 & 19,9 & 22,4 \\
\hline 1 & 90 & 32,4 & & 99 \\
\hline
\end{tabular}


Продолжение табл. 1

\begin{tabular}{|c|c|c|c|c|c|}
\hline 1 & 2 & 3 & 4 & 5 & 6 \\
\hline 3 & 90 & 90 & 52,8 & 38,3 & 91 \\
\hline 5 & 90 & 90 & 69,8 & 37,7 & 123 \\
\hline 7 & 90 & 90 & 89,4 & 31,9 & 126 \\
\hline 1 & 90 & 120 & 46,3 & 28,5 & 90 \\
\hline 3 & 90 & 120 & 57,1 & 38,0 & 112 \\
\hline 5 & 90 & 120 & 67,0 & 38,1 & 128 \\
\hline 7 & 90 & 120 & 90,8 & 30,7 & 131 \\
\hline
\end{tabular}

\section{Результаты и обсуждение}

Дисперсионный анализ и математическое моделирование были осуществлены для каждого выходного параметра процесса получения суберинового связующего от условий щелочного гидролиза бересты.

\section{Степень гидролиза бересты березы}

Экспериментально наблюдаемый характер зависимостей степени гидролиза бересты березы от условий щелочной обработки позволил выбрать факторы для проведения дисперсионного и регрессионного анализов (табл. 2).

Дисперсионный анализ показал, что существенный вклад в суммарную дисперсию степени гидролиза вносят два фактора: концентрация (C) и температура (T). На это указывают высокие значения дисперсионных отношений в строках С и Т. Влияние источника дисперсии на выходной параметр считается статистически значимым, если уровень значимости меньше 0,05 , и это условие выполняется также только для факторов С и $\mathrm{T}$.

Зависимость степени гидролиза бересты березы от переменных факторов процесса щелочной обработки аппроксимирована уравнением регрессии второго порядка

$$
\begin{aligned}
& \mathrm{HD}=-34,59+4,311 \mathrm{C}+0,794 \mathrm{~T}+0,217 \mathrm{P}+0,398 \mathrm{C}^{2}+ \\
& +0,00808 \mathrm{C}-0,00281 \mathrm{CP}-0,0025 \mathrm{~T}^{2}-0,000312 \mathrm{P}^{2} .
\end{aligned}
$$

Был рассчитан коэффициент детерминации $\left(\mathrm{R}^{2}=96,3\right.$ \%), близкий по смыслу к коэффициенту множественной корреляции между выходным параметром (HD) и переменными факторами. Этот коэффициент имеет высокое значение, что может указывать на хорошее качество аппроксимации (максимально возможная величина коэффициента детерминации 100 \%). Более надежной будет оценка $\mathrm{R}_{\mathrm{adj}}{ }^{2} \mathrm{c}$ поправкой на число степеней свободы (последняя строка в табл. 2). В рассматриваемом случае величины $\mathrm{R}^{2}$ и $\mathrm{R}_{\mathrm{adj}}{ }^{2}$ достаточно близки между собой и к 100 \%. Это позволяет говорить об адекватности уравнения (1) результатам наблюдений и использовать его в качестве математической модели изучаемого процесса.

Прогностические свойства уравнения (1) наглядно демонстрируются рис. 1, на котором сравниваются значения выходного параметра $\mathrm{HD}$, наблюдавшиеся в эксперименте (observed), со значениями, прогнозируемыми по модели (predicted). 
Таблица 2. Дисперсионный анализ для степени гидролиза бересты березы

\begin{tabular}{|l|c|c|c|c|c|}
\hline $\begin{array}{c}\text { Источники } \\
\text { дисперсии }\end{array}$ & $\begin{array}{c}\text { Суммы } \\
\text { квадратов }\end{array}$ & $\begin{array}{c}\text { Степени } \\
\text { свободы }\end{array}$ & $\begin{array}{c}\text { Средние } \\
\text { квадраты } \\
\text { сионные } \\
\text { отношения } \\
F\end{array}$ & $\begin{array}{c}\text { Уровни } \\
\text { 3начимости } \\
P\end{array}$ \\
\hline $\mathrm{C}$ & 4532,81 & 1 & 4532,81 & 193,23 & $\mathbf{0 , 0 0 0 0}$ \\
\hline $\mathrm{T}$ & 217,804 & 1 & 217,804 & 9,28 & $\mathbf{0 , 0 0 8 2}$ \\
\hline $\mathrm{P}$ & 63,721 & 1 & 63,721 & 2,72 & 0,1201 \\
\hline $\mathrm{C}^{2}$ & 60,8017 & 1 & 60,8017 & 2,59 & 0,1283 \\
\hline $\mathrm{CT}$ & 2,35225 & 1 & 2,35225 & 0,10 & 0,7559 \\
\hline $\mathrm{CP}$ & 0,497286 & 1 & 0,497286 & 0,02 & 0,8862 \\
\hline $\mathrm{T}^{2}$ & 20,25 & 1 & 20,25 & 0,86 & 0,3675 \\
\hline $\mathrm{P}^{2}$ & 0,230114 & 1 & 0,230114 & 0,01 & 0,9224 \\
\hline $\mathrm{Oc}^{2}$ & 351,879 & 15 & 23,4586 & - & - \\
\hline Общая сумма $^{2}$ & 9533,01 & 23 & - & - & - \\
\hline $\mathrm{R}^{2}$ \% & 96,3 & - & - & - & - \\
\hline $\mathrm{R}_{\text {адј }}^{2}$ с поправкой на число & 94,3 & - & - & - & - \\
\hline
\end{tabular}

Plot of HD

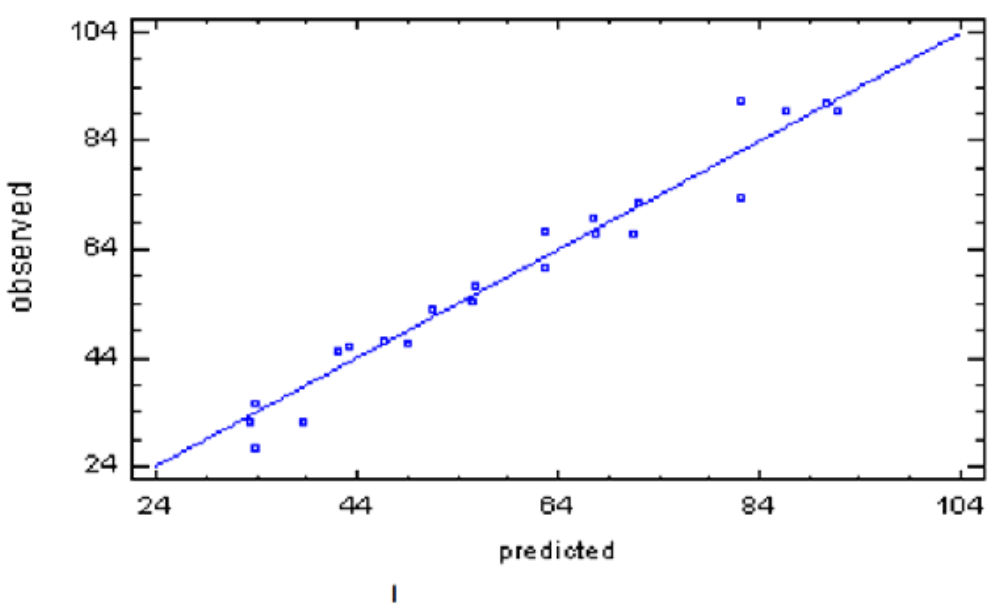

Рис. 1. Результаты наблюдений (точки) против значений выходного параметра HD, прогнозируемых математической моделью

Прямая линия соответствует расчетным (прогнозируемым) значениям HD, точки - результатам наблюдений. Близость «экспериментальных точек» к прямой подтверждает хорошие прогностические свойства уравнения (1).

Математическая модель использована для графического отображения (в виде поверхностей отклика) зависимости степени гидролиза бересты (HD) от переменных факторов процесса щелочной обработки (рис. 2). 


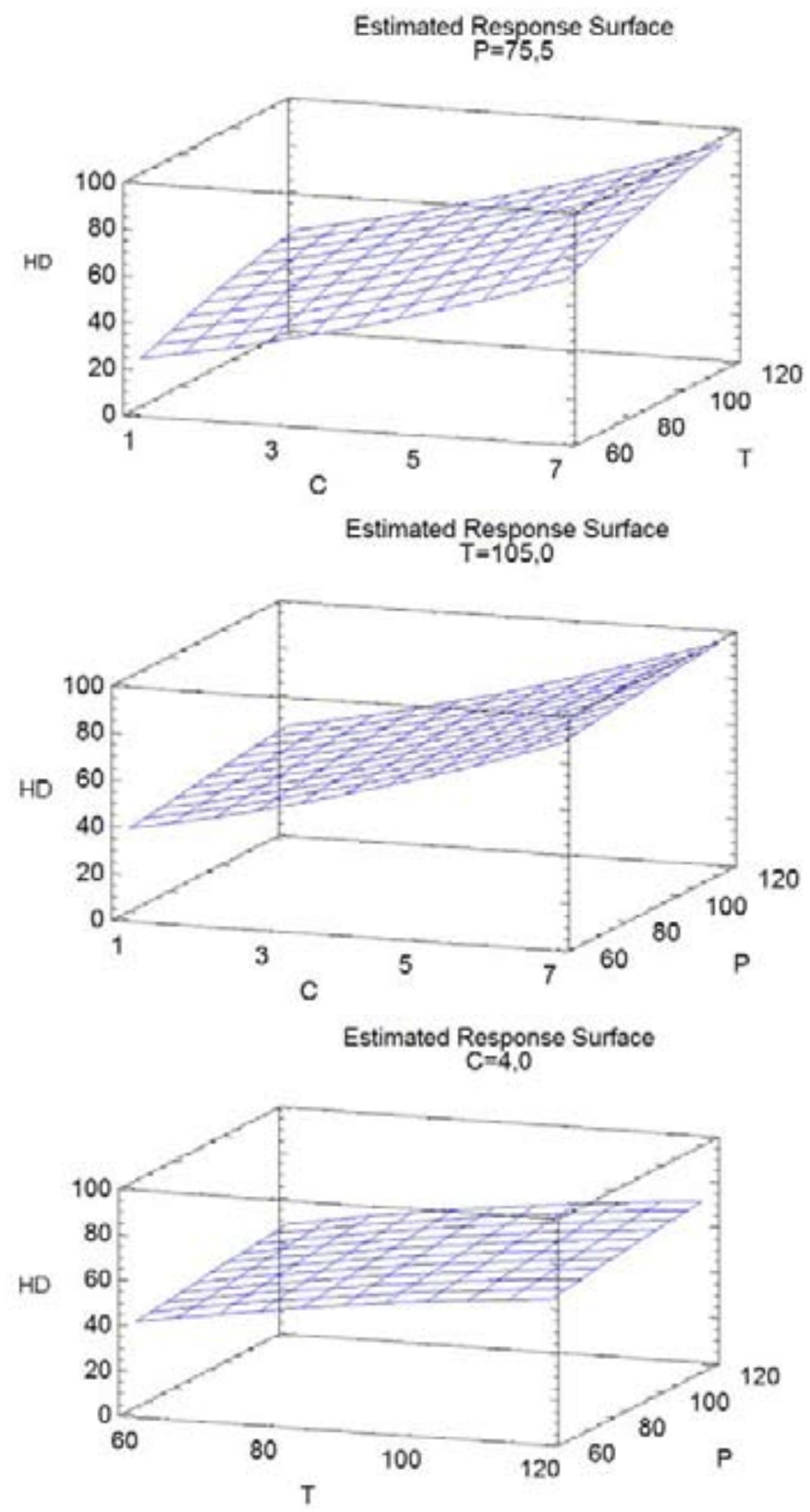

Рис. 2. Поверхности отклика зависимости степени гидролиза от переменных факторов процесса

Все поверхности отклика представляют собой наклонные плоскости или «почти плоскости», т. е. отражают линейные зависимости степени гидролиза от переменных факторов. Этот результат согласуется с рассмотренными выше результатами дисперсионного анализа.

Максимальное прогнозируемое значение $\mathrm{HD}=100$ \% в изученной области факторного пространства достигается согласно вычислениям по математической модели (1) при наибольших значениях независимых переменных, при которых проводились наблюдения, т.е. при концентрации щелочи $\mathrm{C}=7 \%$, температуре $\mathrm{T}=120^{\circ} \mathrm{C}$ и продолжительности $\mathrm{P}=120$ мин.

$$
-261-
$$




\section{Выход суберина}

Результаты дисперсионного анализа указывают на слабое влияние переменных факторов проведения процесса щелочной обработки бересты коры березы на выход суберина (невысокий коэффициент детерминации, низкие эффективности источников дисперсии).

Уравнение регрессии, аппроксимирующее зависимость выхода суберина (V) от переменных факторов, имеет вид

$$
\begin{aligned}
& \mathrm{V}=-6,50+13,9 \mathrm{C}+0,0611 \mathrm{~T}+0,115 \mathrm{P}-1,13 \mathrm{C}^{2}-0,0339 \mathrm{CT}- \\
& -0,0103 \mathrm{CP}+0,00104 \mathrm{~T}^{2}-0,000153 \mathrm{P}^{2} .
\end{aligned}
$$

Рисунок 3 демонстрирует очень большой «разброс» точек вокруг прямой. Характер отклонений точек (равномерное распределение вдоль прямой) позволяет предполагать, что основной причиной сравнительно низкой эффективности модели является так называемый экспериментальный «шум», который складывается из потерь на стадиях осаждения, нейтрализации и промывания суберина.

Графические отображения зависимости выхода суберина (V) от переменных факторов щелочной обработки бересты березы изображены в виде поверхностей отклика на рис. 4.

Следует отметить нелинейную зависимость выхода суберина от концентрации щелочи: наибольшие значения V приходятся на область концентраций $3 . .5$ \%. Этого следовало ожидать, так как только источник дисперсии $\mathrm{C}^{2}$ продемонстрировал высокую эффективность при дисперсионном анализе. Зависимости выхода от температуры и продолжительности близки к линейной зависимости.

Максимальное прогнозируемое значение выхода суберина $(\mathrm{V})=43,8 \%$ в изученной области факторного пространства достигается согласно вычислениям по математической модели

Таблица 3. Дисперсионный анализ для выхода суберина

\begin{tabular}{|l|c|c|c|c|c|}
\hline $\begin{array}{c}\text { Источники } \\
\text { дисперсии }\end{array}$ & $\begin{array}{c}\text { Суммы } \\
\text { квадратов }\end{array}$ & $\begin{array}{c}\text { Степени } \\
\text { свободы }\end{array}$ & $\begin{array}{c}\text { Средние } \\
\text { квадраты }\end{array}$ & $\begin{array}{c}\text { Диспер- } \\
\text { сионные } \\
\text { отношения } F\end{array}$ & $\begin{array}{c}\text { Уровни } \\
\text { значимости } P\end{array}$ \\
\hline $\mathrm{C}$ & 19,7615 & 1 & 19,7615 & 0,98 & 0,3376 \\
\hline $\mathrm{T}$ & 49,8817 & 1 & 49,8817 & 2,48 & 0,1364 \\
\hline $\mathrm{P}$ & 6,52003 & 1 & 6,52003 & 0,32 & 0,5778 \\
\hline $\mathrm{CT}$ & 490,51 & 1 & 490,51 & 24,36 & $\mathbf{0 , 0 0 0 2}$ \\
\hline $\mathrm{CP}$ & 41,4122 & 1 & 41,4122 & 2,06 & 0,1721 \\
\hline $\mathrm{T}^{2}$ & 6,75804 & 1 & 6,75804 & 0,34 & 0,5710 \\
\hline $\mathrm{P}^{2}$ & 3,51563 & 1 & 3,51563 & 0,17 & 0,6820 \\
\hline Остаточная сумма & 0,055 & 1 & 0,055 & 0,00 & 0,9590 \\
\hline Общая сумма & 302,092 & 15 & 20,1394 & - & - \\
\hline $\mathrm{R}^{2}, \%$ & 1086,8 & 23 & - & - & - \\
\hline $\begin{array}{l}\mathrm{R}_{\text {аdј }}^{2} \text { с поправкой } \\
\text { на число степеней } \\
\text { свободы, \% }\end{array}$ & 72,2 & - & - & - & - \\
\hline
\end{tabular}


Plot of $\mathrm{V}$

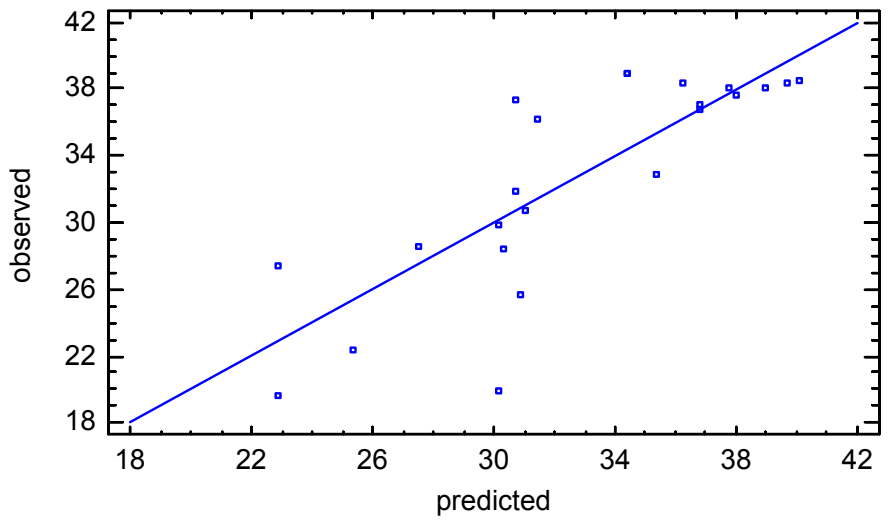

Рис. 3. Результаты наблюдений (точки) против значений выходного параметра V, прогнозируемых математической моделью
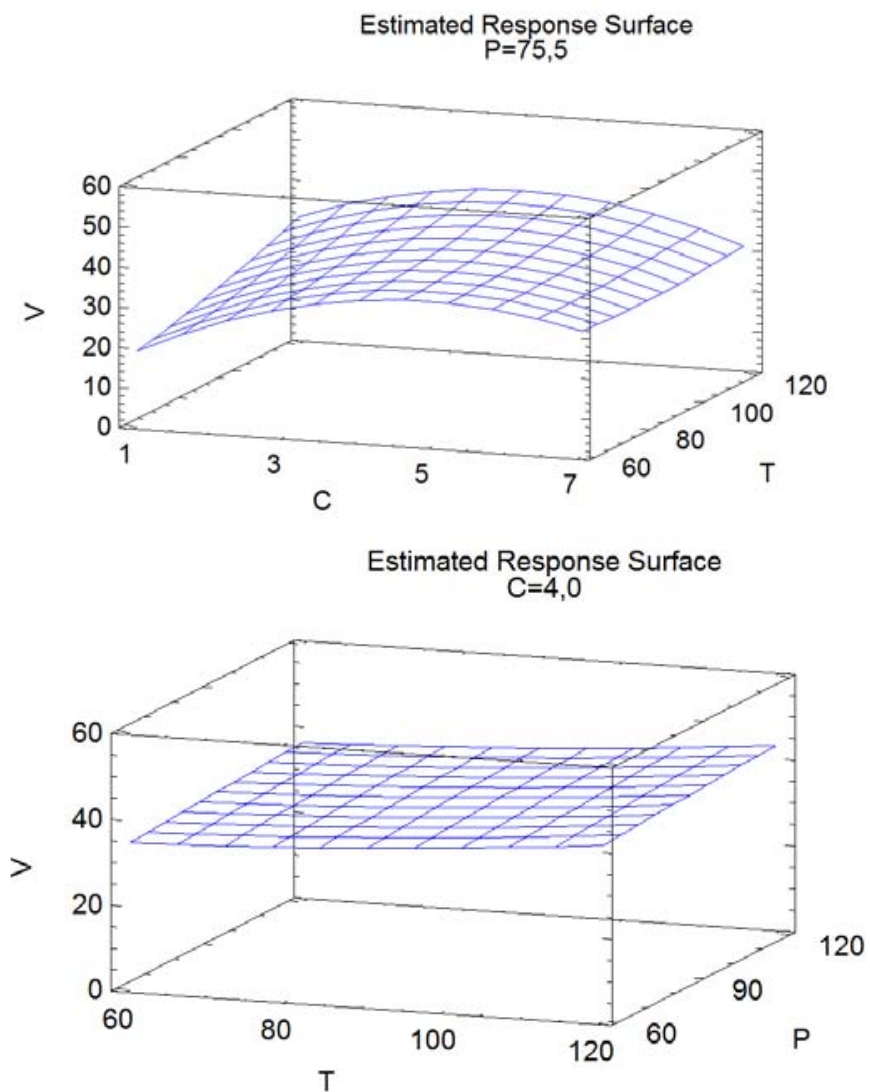

Рис. 4. Поверхности отклика зависимости выхода суберина от переменных факторов процесса 
(2) в точке, соответствующей следующим значениям переменных факторов: концентрация щелочи $3,8 \%$, температура $120^{\circ} \mathrm{C}$, продолжительность процесса 120 мин.

\section{Кислотное число суберинового связующего}

Результаты дисперсионного анализа показали, что только главные (линейные) эффекты вносят существенный вклад в суммарную дисперсию выходного параметра: их эффективность выше 8, уровень значимости меньше 0,05 (табл. 4).

Уравнение регрессии, аппроксимирующее зависимость кислотного числа (К) от переменных факторов, имеет вид

$$
\begin{aligned}
& \mathrm{K}=-1,931+2,420 \mathrm{C}+0,771 \mathrm{~T}+0,3932 \mathrm{P}+0,292 \mathrm{C}^{2}-0,02083 \mathrm{CT}+ \\
& +0,0376 \mathrm{CP}-0,000833 \mathrm{~T}^{2}-0,00132 \mathrm{P}^{2} .
\end{aligned}
$$

Рисунок 5 демонстрирует хорошие прогностические свойства математической модели (3): точки, соответствующие результатам опытов, относительно компактно группируются вокруг прямой.

Зависимости выходного параметра К от независимых переменных представлены поверхностями отклика на рис. 6. Все поверхности имеют форму наклонных плоскостей (или очень близки к плоскостям). Этого следовало ожидать, принимая во внимание результаты дисперсионного анализа. Отметим, что все поверхности отклика выходных параметров НD и К не только имеют одинаковую форму, но и наклонены к горизонтальной плоскости под одинаковыми углами (т.е. одинаково ориентированы в факторном пространстве). Это указывает на существование причинно-следственной связи между степенью гидролиза бересты березы и кислотным числом получаемого суберинового связующего.

Таблица. 4. Дисперсионный анализ для кислотного числа суберинового связующего

\begin{tabular}{|l|c|c|c|c|c|}
\hline $\begin{array}{c}\text { Источники } \\
\text { дисперсии }\end{array}$ & $\begin{array}{c}\text { Суммы } \\
\text { квадратов }\end{array}$ & $\begin{array}{c}\text { Степени } \\
\text { свободы }\end{array}$ & $\begin{array}{c}\text { Средние } \\
\text { квадраты }\end{array}$ & $\begin{array}{c}\text { Диспер- } \\
\text { сионные } \\
\text { отношения } F\end{array}$ & $\begin{array}{c}\text { Уровни } \\
\text { значимости } P\end{array}$ \\
\hline $\mathrm{C}$ & 2003,67 & 1 & 2003,67 & 55,57 & $\mathbf{0 , 0 0 0 0}$ \\
\hline $\mathrm{T}$ & 630,375 & 1 & 630,375 & 17,48 & $\mathbf{0 , 0 0 0 8}$ \\
\hline $\mathrm{P}$ & 301,287 & 1 & 301,287 & 8,36 & $\mathbf{0 , 0 1 1 2}$ \\
\hline $\mathrm{C}^{2}$ & 32,6667 & 1 & 32,6667 & 0,91 & 0,3563 \\
\hline $\mathrm{CT}$ & 15,625 & 1 & 15,625 & 0,43 & 0,5203 \\
\hline $\mathrm{CP}$ & 89,1571 & 1 & 89,1571 & 2,47 & 0,1367 \\
\hline $\mathrm{T}^{2}$ & 2,25 & 1 & 2,25 & 0,06 & 0,8061 \\
\hline $\mathrm{P}^{2}$ & 4,10227 & 1 & 4,10227 & 0,11 & 0,7406 \\
\hline Остаточная сумма & 540,876 & 15 & 36,0584 & - & - \\
\hline Обшая сумма $^{2}$ & 7947,83 & 23 & - & - & - \\
\hline $\begin{array}{l}\mathrm{R}_{\text {аdј }}^{2} \text { с поправкой } \\
\text { на число степеней } \\
\text { свободы, \% }\end{array}$ & 93,2 & - & - & & - \\
\hline
\end{tabular}




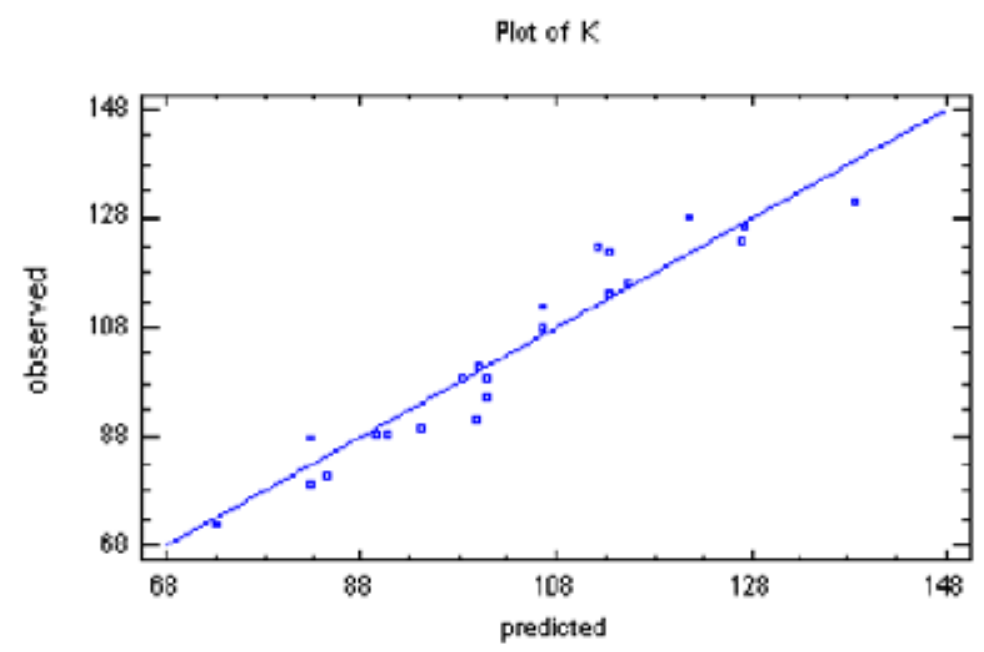

Рис. 5. Результаты наблюдений (точки) против значений выходного параметра К, прогнозируемых математической моделью

Максимальное значение кислотного числа $(K)=152$ мг $\mathrm{NaOH} /$ в в пределах изученного факторного пространства согласно математической модели (3) прогнозируется при следующих условиях: концентрация щелочи 7,0 \%, температура гидролиза $120^{\circ} \mathrm{C}$, продолжительность гидролиза 120 мин.

Оптимизащия процесса получения суберинового связующего

щелочным гидролизом бересты коры березы

С учетом существования ограничения на величину кислотного числа, а также отмеченной выше связи между степенью гидролиза бересты березы и кислотным числом задача формулируется следующим образом: в пределах изученного факторного пространства найти условия, обеспечивающие достижение максимально полного выделения суберина с кислотным числом не выше 110 мг $\mathrm{NaOH} / г$. Эта задача квадратичного программирования записывается следующим образом:

целевая функция

$\mathrm{V} \rightarrow \max ; \mathrm{V}$ задается уравнением (2);

ограничения на другие выходные параметры

$\mathrm{K} \leq 110$; К задается уравнением (3);

ограничение области поиска

$$
\begin{aligned}
& 1 \leq \mathrm{C} \leq 7 ; \\
& 60 \leq \mathrm{T} \leq 120 ; \\
& 60 \leq \mathrm{P} \leq 120 .
\end{aligned}
$$

Решение задачи оптимизации процесса получения субериновых связующих щелочным гидролизом бересты коры березы с использованием градиентного метода дало следующие результаты:

концентрация щелочи $(\mathrm{C})=3,1 \%$;

$$
-265-
$$



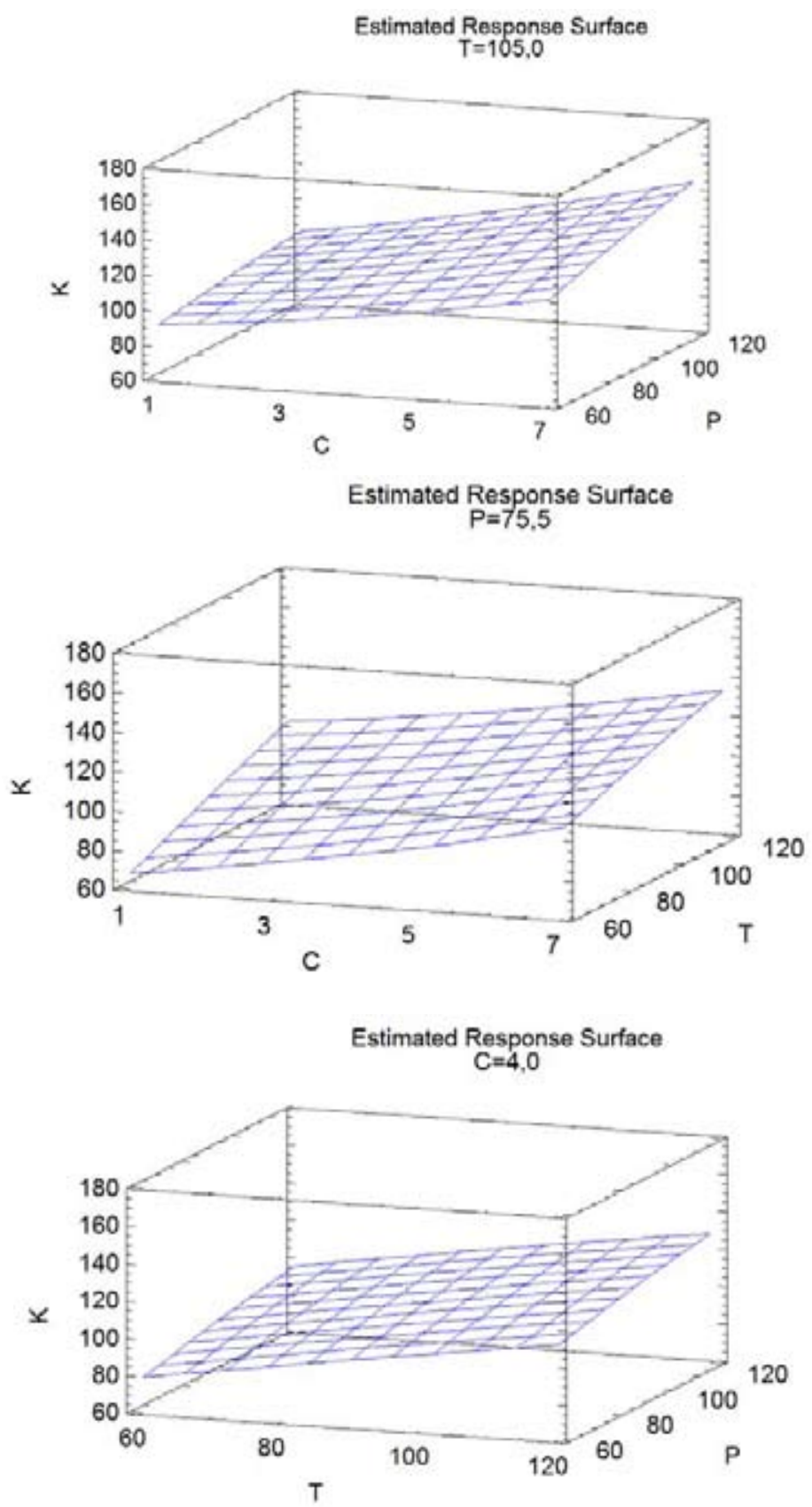

Рис. 6. Поверхности отклика зависимости кислотного числа от переменных факторов процесса

температура гидролиза $(\mathrm{T})=88^{\circ} \mathrm{C}$;

продолжительность гидролиза $(\mathrm{P})=120$ мин;

при этом степень гидролиза бересты достигает (HD) 55,8 \%, выход суберина составляет (V) 37,8 \% и кислотное число $(\mathrm{K})=106,7$ мг $\mathrm{NaOH} / г$. 


\section{Заключение}

В результате дисперсионного анализа экспериментальных данных получены регрессионные уравнения, которые можно использовать в качестве математической модели, адекватно описывающей влияние условий щелочного гидролиза бересты коры березы на выход и кислотное число суберинового связующего.

На основании математического моделирования и решения задачи оптимизации процесса были определены условия щелочного гидролиза бересты коры березы с максимальным выходом суберинового связующего 37,8 \% мас.: концентрация щелочи 3,1 \% мас., температура гидролиза $88^{\circ} \mathrm{C}$, продолжительность гидролиза 120 мин.

\section{Список литературы}

1. Савельев М.С. Биотопливо на основе древесных отходов // Российский внешнеэкономический вестник. 2007. №12. C. 4-9.[Saveliev M.S Biofuels based on wood waste // Russian External Gazette 2007. No 12. P. 4-9 (in Russ.)].

2. Sikkema R., Steiner M., Junginger M., Hiegl W., Hansen M.T., Faaij A. The European wood pellet markets: current status and prospects for 2020 // Biofuels, Bioproducts and Biorefining. 2011. V 5, No 3. P. 250-278.

3. Назаров В.И., Булатов И.А., Макаренков Д.А. Особенности разработки процесса прессового гранулирования биотоплива на основе древесных и растительных отходов // Химическое и нефтегазовое машиностроение. 2009. № 2. C. 35-39. [Nazarov V.I., Bulatov I.A., Makarenkov D.A. Features of the development process of press granulation of biofuels based on wood and plant waste // Chemical and Petroleum Engineering 2009. № 2. P. 35-39. (in Russ.)].

4. Гомонай М.В. Производство топливных брикетов. Древесное сырье, оборудование, технологии, режимы работы: монография. М.: ГОУ ВПО МГУЛ, 2006. 68 с.[Gomonay M.V. Production of fuel briquettes. Wood raw materials, equipment, technology, operation modes: a monograph. M .: GOU VPO MGUL, 2006. 68 p.(in Russ.)].

5. Boris N. Kuznetsov, Irina G. Sudakova, Alain Celzard, Natalia V. Garyntseva, Natalia M. Ivanchenko, Andrey V. Petrov Binding properties of lignins obained at oxidative catalytic delignification of wood and straw // Journal of Siberian Federal University. Chemistry. 2011. V.1(4). P.3-10.

6. Кислицын А.Н. Экстрактивные вещества бересты: выделение, состав, свойства, применение // Химия древесиныл. 1994. №3. C.10-19.[Kislitsyn A.N. Extractive substances bark: extraction, composition. Properties, application // Chemical wood. 1994. No3. P. 10-19.(in Russ.)].

7. Paula C.R.O. Pinto, Andreia F. Sousa, Armando J.D. Silvestre, Carlos Pascoal Neto, Alessandro Gandini, Christer Eckerman, Bjarne Holmbom. Quercus suber and Betula pendula outer barks as renewable sources of oleochemicals: A comparative study // Iindustrial Crops and Products. 2009. V.29. P. 126-132.

8. Alessandro Gandini, Carlos Pascoal Neto, Armando J.D. Silvestre. Suberin: A promising renewable resource for novel macromolecular materials // Progress in Polymer Science. 2006. V. 31. P. 878-892.

9. Судакова И.Г., Гарынцева Н.В., Иванов И.П., Кузнецов Б.Н. Выделение и применение суберина из бересты коры березы // Журнал СФУ. Химия. 2012. № 2 (5). С. 168-177. [Sudakova I.G., 
Garyntseva N.V., Ivanov I.P., Kuznetsov B.N. Isolation and use of suberin from birch bark // Journal of Siberian Federal University. Chemistry. 2012. No 2 (5). P. 168-177.(in Russ.)].

10. Судакова И.Г., Кузнецов Б.Н., Гарынцева Н.В. Изучение процесса выделения субериновых веществ из бересты березовой коры // Химия растительного сырья. 2008. №1. С. 41-44. [Sudakova I.G., Kuznetsov B.N., Garyntseva N.V. Study of the process of suberin isolation from the birch bark outer layer // Chemistry of Plant Raw Materialis. 2008. No 1. P. 41-44. (in Russ.)].

11. Irina G. Sudakova, Boris N. Kuznetsov, Natalia V. Garyntseva, Nina I. Pavlenko, Natalia M. Ivanchenko Functional and thermal analysis of suberin isolated from birch bark // Journal of Siberian Federal University. Chemistry. 2008. V.4(1). P. 355-362.

12. Судакова И.Г., Кузнецов Б.Н., Иванов И.П., Иванченко Н.М. Получение огнезащитных составов на основе суберина коры березы // Вестник КрасГУ. Естественные науки. 2005. С. 101105. [Sudakova I.G., Kuznetsov B.N., Ivanov I.P., Ivanchenco N.M. The obtaining of wood fire-proof composition on the base of birch bark // Vestnik Krasnoyarsk State University. Natural Science. 2005. P.101-105. (in Russ.)].

13. Судакова И.Г., Кузнецов Б.Н., Иванов И.П., Иванченко Н.М. Получение пленкообразующих материалов из суберина коры березы повислой // Химия растительного сырья. 2004. № 1. C.31-34. [Sudakova I.G., Kuznetsov B.N., Ivanov I.P., Ivanchenco N.M. Film-forming materials production from birch bark suberin // Chemistry of Plant Raw Materialis. 2004. No 1. P. 31-34. (in Russ.)].

14. Судакова И.Г., Гарынцева Н.В., Кузнецов Б.Н. Получение древесных плитных материалов с использованием связующих на основе суберина березовой коры // Химия растительного сырья. 2011. № 3. C.65-68. [Sudakova I.G., Garyntseva N.V., Kuznetsov B.N. Production of wood fiber boards with the use of suberin-derived binding agents // Chemistry of Plant Raw Materialis. 2011. No 3. P. 65-68. (in Russ.)].

15. Судакова И.Г., Иванченко Н.М., Кузнецов Б.Н. Получение древесных топливных брикетов с использованием связующих из суберина березовой коры // Химия растительного сblрья. 2008. №2. C. 31-34. [Sudakova I.G., Ivanchenko N.M., Kuznetsov B.N. Production of wood briquettes using binders from birch bark suberin // Chemistry of plant raw materials 2008. No2. P. 3134. (in Russ)].

16. Pat. WO 2010093320 (A1) IPC C07C51/42; C07C59/305; C07C59/58 The method for separating from suberin and/or cutin containing plants, a solid and/or oil fraction enriched in cis-9,10-epoxy18-hydroxyoctadecanoic acid / T. Inversen, H.Nilsson, A. Olsson; priority 13.02.2009; publication 19.08.2010.

17. Пен Р.3. Планирование эксперимента в Statgraphics Centurion. Красноярск, 2014. 293 с. [Pen R.Z. Planning of experiment in Statgraphics Centurion. Krasnoyarsk, 2014. 293 p. (in Russ.)]

18. Курицкий Б.Я. Поиск оптимальных решений средствами Excel в примерах. СПб., 1997. 384 c. [Kuritskiy B.J. The search for optimal solutions by means of Excel in the examples. St. Petersburg, 1997. 384 p. (in Russ.)]. 